\title{
Relapsing Haemothorax as an Unusual Presentation of Primary Angiosarcoma of the Spleen
}

\author{
Alba Bergas ${ }^{1}$, Francesc Escrihuela-Vidal ${ }^{1}$, Davinia Fernández Calvo ${ }^{2}$, Olga Capdevila Pons ${ }^{1}$, Xavier Corbella $^{1,3}$ \\ ${ }^{1}$ Department of Internal Medicine, Hospital Universitari de Bellvitge, Bellvitge Biomedical Research Institute-IDIBELL, L'Hospitalet de Llobregat, Spain \\ ${ }^{2}$ Department of Pathology, Hospital Universitari de Bellvitge, Bellvitge Biomedical Research Institute-IDIBELL, L'Hospitalet de Llobregat, Spain \\ ${ }^{3}$ School of Medicine, Universitat Internacional de Catalunya, Barcelona, Spain
}

\section{Doi: 10.12890/2020_001944- European Journal of Case Reports in Internal Medicine - ( ) EFIM 2020}

Received: $18 / 08 / 2020$

Accepted: $25 / 08 / 2020$

Published: $29 / 10 / 2020$

How to cite this article: Bergas A, Escrihuela-Vidal F, Fernández Calvo D, Capdevila Pons O, Corbella X. Relapsing haemothorax as an usual presentation of primary angiosarcoma of the spleen. EJCRIM 2020;7: doi:10.12890/2020_001944.

Conflicts of Interests: The Authors declare that there are no competing interests.

Acknowledgements: We thank CERCA Programme/Generalitat de Catalunya for institutional support.

This article is licensed under a Commons Attribution Non-Commercial 4.0 License

\section{ABSTRACT}

Spontaneous haemothorax $(\mathrm{SH})$ is a rare subcategory of haemothorax that involves the accumulation of blood within the pleural space in the absence of trauma. SH especially occurs in middle-aged or elderly patients, but data are usually limited to case reports and case series. Coagulopathy, aneurysm or aortic dissection, Rendu-Osler-Weber syndrome and malignancy have to be considered among the causes of SH. Aim: We describe a case of primary angiosarcoma of the spleen presenting as relapsing haemothorax.

Results: An 81-year-old woman was referred to our hospital because of a 2-month history of relapsing haemothorax after the performance of urgent splenectomy due to active bleeding from large spleen cysts. No evidence of neoplasm was seen after pathological examination of the spleen. On admission, left haemothorax and 2 new cystic masses in the thoracic wall were documented, both in close relation to the scars of previous surgery and chest tubes. After excision of 1 mass, histological examination revealed angiosarcoma, and a final diagnosis of primary angiosarcoma of the spleen with postsurgical metastatic dissemination to the thorax and pleura was made.

Conclusion: Primary angiosarcoma should be included in the differential diagnosis of haemorrhagic spleen cysts. Clinical diagnosis and management usually requires splenectomy, but it should be carefully planned in order to avoid local metastatic dissemination or haematogenous spread of the tumour.

\section{LEARNING POINTS}

- The differential diagnosis of spleen cysts must include malignant conditions.

- Despite its absolute rarity, angiosarcoma is considered the most common primary non-haematolymphoid splenic malignancy and has a poor prognosis.

- Splenectomy is usually required for a prompt diagnosis of primary angiosarcoma of the spleen, but it needs to be performed with extreme caution to avoid metastatic dissemination.

\section{KEYWORDS}

Spleen, angiosarcoma, haemothorax 


\section{INTRODUCTION}

Primary malignant processes of the spleen are extremely rare and commonly mistaken for benign vascular or malignant non-vascular tumours due to highly variable histology. Despite its absolute rarity, angiosarcoma is considered the most common primary non-haematolymphoid splenic malignancy ${ }^{[1,2]}$. Despite diagnosis and clinical management that includes splenectomy in most cases, prognosis is very poor because of early multifocal metastasis.

\section{CASE DESCRIPTION}

An 81-year-old woman was referred to our hospital because of relapsing haemothorax after urgent splenectomy. Two months before this admission, the patient was hospitalized at another hospital because of a 4-month history of fatigue and non-quantified weight loss. Blood tests showed iron deficiency anaemia and elevated acute-phase reactants. A computed tomography (CT) scan showed splenomegaly with 2 large cystic lesions $(10 \times 8 \mathrm{~cm}$ and $5 \times 5 \mathrm{~cm})($ Fig. $1 \mathrm{~A})$.

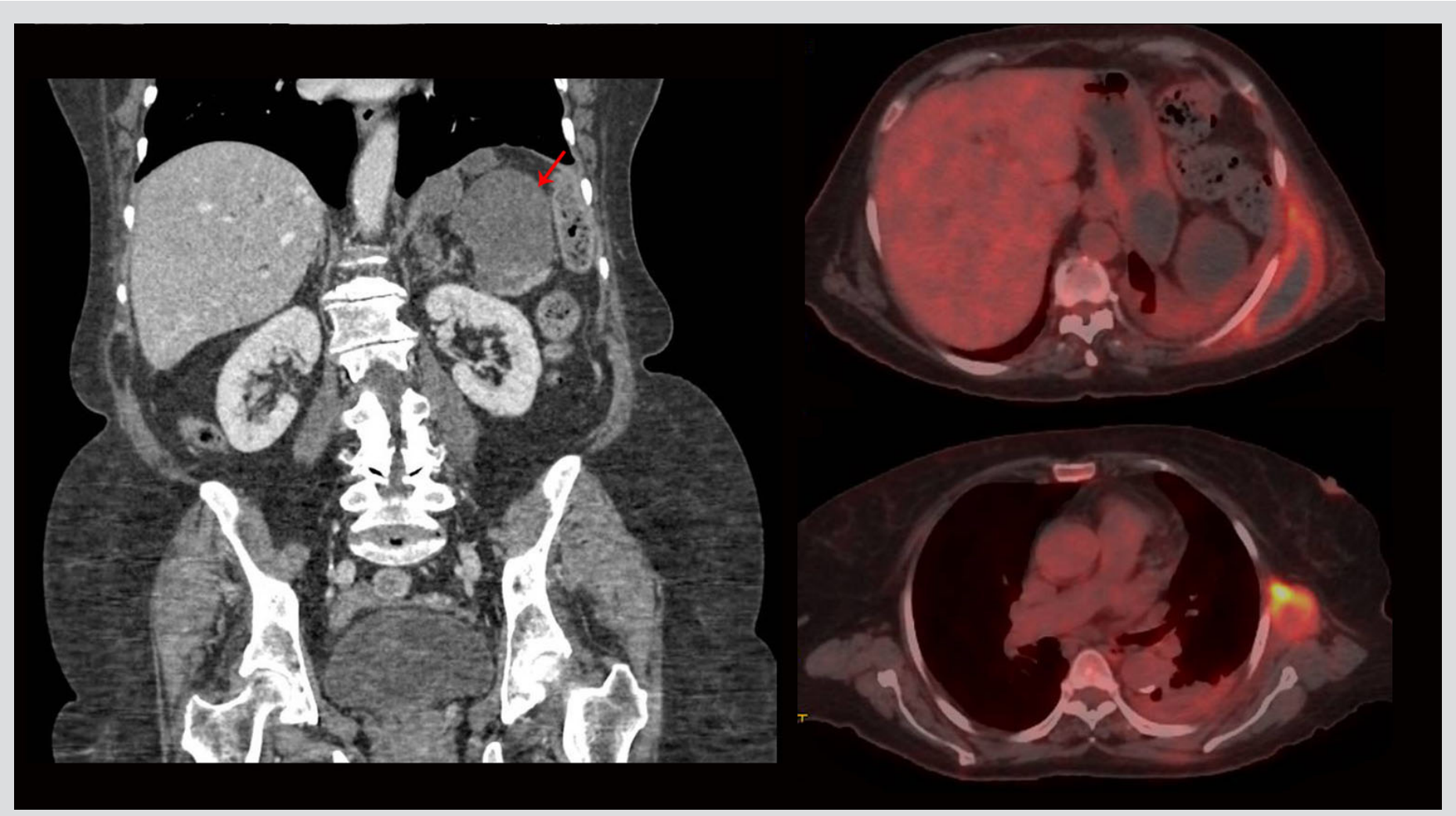

Figure 1. A. CT showed 2 splenic cystic lesions with signs of active bleeding. B. Metabolic activity was present in all masses on PET-CT

Urgent splenectomy was indicated because clinical and radiological signs suggested active bleeding of 1 of the spleen cysts. Pathological examination of the spleen described the presence of pseudocysts containing necrotic and haemorrhagic material, with poorly defined margins, acute and chronic inflammatory infiltrates in the splenic parenchyma and no evidence of neoplasm. Soon after the surgery, the patient was discharged. However, 2 months later she was readmitted due to progressive shortness of breath and pleuritic chest pain due to extensive left postsurgical pleural effusion. A chest tube was then placed, showing pleural haemorrhagic exudation, with no malignant cells or microorganisms. The subsequent clinical course was significant for progressive anaemia and spiking fever, and a new haematic collection was detected in the thoracic surgical site. At that time, the patient was referred to our institution. On arrival, physical examination showed 2 new subcutaneous and painful masses of $5 \mathrm{~cm}$ each in the left posterior thoracic wall, both in close relation to the scars of previous surgery and chest tube introduction. Laboratory tests revealed severe anaemia and increased acute-phase reactants. A CT scan confirmed the extensive left pleural effusion and the 2 cystic masses described earlier, but also showed 2 additional subpleural cystic nodules in the left thorax and $25 \times 3 \mathrm{~cm}$ lobulated cystic masses in the greater curvature of the stomach. 
The radiological characteristics of the masses were similar to the spleen cysts from the splenectomy performed during the patient's first admission. Positron emission tomography-CT (PET-CT) demonstrated 18F-fluorodeoxyglucose uptake in all masses (Fig. 1B), this being maximal in the subcutaneous thoracic cysts. The analysis of pleural fluid showed haemothorax with no evidence of malignant cells or infection. A chest tube was then placed in the left pleural space and intrapleural urokinase was administered. Coagulopathy, autoimmunity and infectious diseases and agents such as tuberculosis, Echinococcus spp. and amoebae were also ruled out. An ultrasound-guided biopsy of 1 of the subcutaneous thoracic masses showed atypical cells with no other findings. Unfortunately, the general condition of the patient worsened rapidly and masses enlarged with progression to severe anaemia, requiring repeated blood transfusions. At that time, 1 of the thoracic masses was excised by a plastic surgeon, giving a diagnosis of angiosarcoma at histological examination (Fig. 2). A final diagnosis of primary splenic angiosarcoma with thoracic and pleural metastatic postsurgical invasion was then made. The patient was finally discharged home for palliative care follow-up, since chemotherapy and radiotherapy could not be indicated due to persistent cytopenia and infectious complications. Three months later, the patient died.

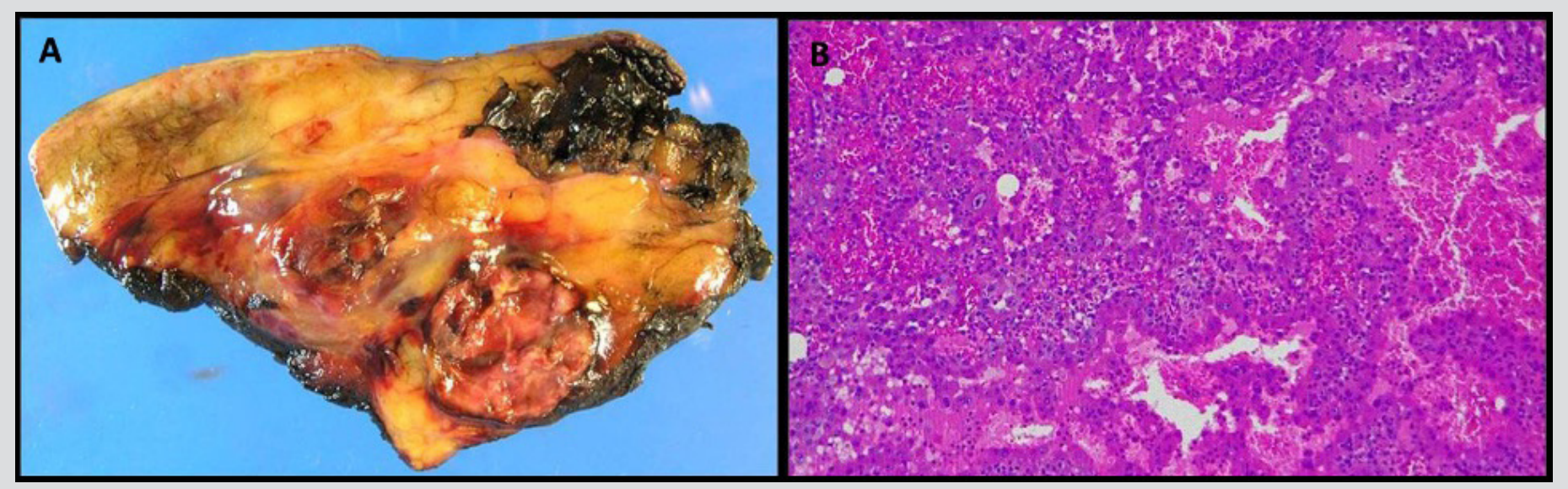

Figure 2. Pathological examination of the cyst. 2A (macroscopy): subcutaneous haemorrhagic lesion with cystic and necrotic areas. 2B (microscopy): vascular cavities covered with an endothelium of atypical epithelioid cells

\section{DISCUSSION}

Angiosarcoma is a rare malignant vascular tumour that accounts for $<2 \%$ of all sarcomas. It usually occurs in middle-aged and elderly patients, and symptoms are closely related to the tumour location, the most common being the skin, heart, liver, spleen, bone and GI tract. Spontaneous haemorrhagic pleural effusion is directly related to pleural invasion from the primary tumour site, and it usually has a bloody appearance with a high haematocrit value and also contains neoplastic cells.

No more than 300 cases of primary angiosarcoma of the spleen have been reported to date, with most of these diagnosed between the fifth and sixth decades of life, with a slight male preponderance. Interestingly, benign splenic tumours such as haemangiomas have been suggested to be a precursor to splenic angiosarcoma ${ }^{[1,2]}$, as well as other environmental and occupational carcinogens ${ }^{[3]}$. The usual symptoms at onset include fatigue, weakness, anorexia, weight loss, abdominal pain ${ }^{[2]}$, and occasionally, fatal haemorrhagic shock due to spontaneous splenic rupture $^{[4-6]}$. The differential diagnosis should consider benign splenic haemangioma and malignant lymphoma, and diagnostic tests should include histological analysis of surgical specimens by biopsy or splenectomy ${ }^{[7-9]}$.

Similar to other forms of angiosarcoma, primary angiosarcoma of the spleen commonly has early multifocal metastasis to the liver, lungs, lymph nodes, bone and ovaries through haematogenous spread. Characteristically, splenic tumour rupture with haemorrhagic shock facilitates peritoneal dissemination of the tumour and confers a very poor prognosis ${ }^{[10,11]}$. Early splenectomy is the preferred therapeutic option, improving outcomes in both localized and metastatic disease, especially if performed before spontaneous splenic rupture ${ }^{[12-14]}$. Although unusual, local metastatic recurrence after surgery has also been described, as occurred in our case. Concurrent chemotherapy has been proposed, but with no specific and effective regimens described to date ${ }^{[14-18]}$. Local radiotherapy has been also used as a haemostatic measure. Nevertheless, the current average survival time is usually less than 6 months after diagnosis ${ }^{[2]}$. 
In conclusion, a comprehensive approach should be carefully developed when studying splenic cystic masses, including consideration of infectious, vascular, autoimmune or malignant diseases. We report the case of an 81-year-old woman with relapsing haemothorax as the unusual presentation of primary angiosarcoma of the spleen. In our case, haemothorax was caused by local metastatic invasion and dissemination from the primary splenic location to the thoracic wall and pleural space, probably after an accidental rupture of the spleen during splenectomy. Therefore, although splenectomy is indicated in most cases of primary angiosarcoma of the spleen, it should be carefully performed in order to avoid metastatic dissemination of the tumour, as this can seriously worsen the already bad prognosis.

\section{REFERENCES}

1. Falk S, Krishnan J, Meis JM. Primary angiosarcoma of the spleen. A clinicopathologic study of 40 cases. Am J Surg Pathol 1993;17:959-970.

2. Neuhauser TS, Derringer GA, Thompson LD, Fanburg-Smith JC, Miettinen M, Saaristo A, et al. Splenic angiosarcoma: a clinicopathologic and immunophenotypic study of 28 cases. Mod Pathol 2000;13(9): 978-987.

3. Naka N, Ohsawa M, Tomita Y, Kanno H, Uchida A, Aozasa K. Angiosarcoma in Japan. A review of 99 cases. Cancer 1995;75(4):989-996.

4. Rosenblatt P, Koka R, Chen Q, Papadimitriou JC, Sausville EA, Emadi A. Schistocytes, echinocytes, iron deficiency anemia, and thrombocytopenia-hematologic manifestations of splenic angiosarcoma. Arch Iran Med 2013;16(10):602-605.

5. Aqil B, Green LK, Lai S. Primary splenic angiosarcoma associated with anemia, leukocytosis and thrombocytopenia. Ann Clin Lab Sci 2014;44(2):217-221.

6. Raffel S, Hildebrandt B, Grieser C, Pahl S, Sturm I. Thrombocytopenia as first manifestation of splenic angiosarcoma. Ann Hematol 2010;89:109-110.

7. Thompson WM, Levy AD, Aguilera NS, Gorospe L, Abbott RM. Angiosarcoma of the spleen: imaging characteristics in 12 patients. Radiology 2005;235:106-115.

8. Delacruz V, Jorda M, Gomez-Fernandez C, Benedetto P, Ganjei P. Fine-needle aspiration diagnosis of angiosarcoma of the spleen: a case report and review of the literature. Arch Pathol Lab Med 2005;129(8):1054-1056.

9. Ferrozzi F, Bova D, Draghi F, Garlaschi G. CT findings in primary vascular tumors of the spleen. AJR Am J Roentgenol 1996:166:1097-1101.

10. Duan YF, Jiang Y, Wu CX, Zhu F. Spontaneous rupture of primary splenic angiosarcoma: a case report and literature review. World J Surg Oncol $2013 ; 11: 53$.

11. Özcan B, Çevener M, Kargı AO, Dikici H, Yıl dız A, Özdo?an M, et al. Primary splenic angiosarcoma diagnosed after splenectomy for spontaneous rupture. Turk J Surg 2018;34(1):68-70.

12. Montemayor P, Caggiano V. Primary hemangiosarcoma of the spleen associated with leukocytosis and abnormal spleen scan. Int Surg 1980;65:369-373.

13. Hsu JT, Chen HM, Lin CY, Yeh CN, Hwang TL, Jan YY, et al. Primary angiosarcoma of the spleen. J Surg Oncol 2005;92:312-316.

14. Hara T, Tsurumi H, Kasahara S. Long-term survival of a patient with splenic angiosarcoma after resection, high-dose chemotherapy, and autologous peripheral blood stem cell transplantation. Intern Med 2010;49:2253-2257.

15. Ferreira BP, Rodler ET, Loggers ET, Pollack SM, Jones RL. Systemic therapy in primary angiosarcoma of the spleen. Rare Tumors 2012;4(4):178-180.

16. Rupolo M, Berretta M, Buonadonna A, Stefanovski P, Bearz A, Bertola G, et al. Metastatic angiosarcoma of the spleen. A case report and treatment approach. Tumori 2001;87:439-443.

17. Zietz C, Rossle M, Haas C, Sendelhofert A, Hirschmann A, Stürzl M, et al. MDM-2 oncoprotein overexpression, p53 gene mutation, and VEGF up-regulation in angiosarcomas. Am J Pathol 1998;153:1425-1433.

18. Italiano A, Cioffi A, Penel N, Levra MG, Delcambre C, Kalbacher E, et al. Comparison of doxorubicin and weekly paclitaxel efficacy in metastatic angiosarcoma. Cancer 2012;118:3330-3336. 